\title{
New Wave Solutions of Time Fractional Kadomtsev-Petviashvili Equation Arising in the Evolution of Nonlinear Long Waves of Small Amplitude
}

\author{
Hülya DURUR ${ }^{1 *} \mathbb{D}$, Orkun TAŞBOZAN ${ }^{2}$, Ali KURT ${ }^{3}$, Mehmet ŞENOL ${ }^{4}$
}

${ }^{1}$ Department of Computer Engineering, Faculty of Engineering, Ardahan University, Ardahan, TURKEY. ${ }^{2}$ Department of Mathematics, Faculty of Science and Art, Hatay Mustafa Kemal University, Hatay, TURKEY.

${ }^{3}$ Department of Mathematics, Faculty of Science and Art, Pamukkale University, Denizli, TURKEY.

${ }^{4}$ Department of Mathematics, Faculty of Science and Art, Nevsehir Haci Bektas Veli University, Nevsehir, TURKEY.

Geliş / Received: 27/11/2018, Kabul / Accepted: 13/05/2019

\begin{abstract}
The main aim of this paper is to obtain the travelling wave solutions of fractional Kadomtsev-Petviashvili(KP) Equation where the derivative is in conformable sense. For this aim the sub equation method is used with computer software called Mathematica. Then, solutions are investigated through the graphical representation for different cases of $\alpha$.
\end{abstract}

Keywords: Kadomtsev-Petviashvili Equation, Sub-Equation Method, Wave Solution; Conformable Fractional Derivative.

Küçük Genlikli Lineer Olmayan Uzun Dalgaların Gelişiminde Türeyen Zaman Kesirli KadomtsevPetviashvili Denkleminin Yeni Dalga Çözümleri

$\ddot{O} \mathbf{z}$

$\mathrm{Bu}$ makalenin asıl amacı, türevleri conformable cinsinden olan kesirli Kadomtsev-Petviashvili (KP) denkleminin dalga çözümlerini bulmaktır. Bu amaç için alt denklem metodu, Mathematica bilgisayar programı ile birlikte kullanılmıştır. Daha sonra elde edilen çözümlerin $\alpha^{\prime}$ nın değişik değerleri için grafiksel gösterimleri verilmiştir.

Anahtar Kelimeler: Kadomtsev-Petviashvili denklemi, Alt-denklem metodu, Dalga çözümü, Conformable kesirli türev.

\section{Introduction}

Nonlinear evolution equations (NLEEs) describe lots of natural phenomena in the fields of science, particularly in physics, engineering, chemistry and finance. Therefore, a diverse group of scientists are paying great attention to acquire the exact solutions of NLEEs [30,31]. For this purpose many different analytical methods are used such as the hyperbolic function method, Hirota method, new auxiliary equation method, the backland transformation, the modified simple equation method, the auxiliary equation method, algebraic method, extended simplest equation method, the sine- 
Gordon expansion method and improved generalized Jacobi elliptic function method [1-14].

Fractional calculus is the best tool to explain the complexity and nonlinearity of the nature. An arising interest to this subject grows day by day. To state the events that occurs in the different branches of science and technology, there are a few different definitions of derivatives and integrals with arbitrary order. Although each of them differs from the other one, generally these definitions include integral form such as Riemann-Liouville and Caputo. This integral form makes the definitions harder to understand and the calculations to obtain the analytical or approximate solutions of the equations become more complicated. In addition it is understood that these definitions have some flaws. For instance

1. The fractional derivative of a constant does not equals zero in Riemann-Liouville sense.

2. The formula for the derivative of the product of two functions is not satisfied by both Riemann-Liouville and Caputo derivative.

$$
D_{a}^{\alpha}(f g)=g D_{a}^{\alpha}(f)+f D_{a}^{\alpha}(g) .
$$

3. The formula for the derivative of the quotient of two functions is not satisfied by both Riemann-Liouville and Caputo derivative.

$$
D_{a}^{\alpha}\left(\frac{f}{g}\right)=\frac{f D_{a}^{\alpha}(f)-g D_{a}^{\alpha}(g)}{g^{2}} .
$$

4. Both Riemann and Caputo derivative do not satisfy the chain rule.

$$
D_{a}^{\alpha}(f \circ g)(t)=f^{\alpha}(g(t)) g^{\alpha}(t) .
$$

5. The property $D^{\alpha} D^{\beta}=D^{\alpha+\beta}$ is not satisfied by both Riemann-Liouville and Caputo derivative in general.
6. It is supposed that the function $f$ is differentiable in Caputo sense.

To overcome this flaws Khalil et. al. [1] expressed applicable definition of noninteger order differentiation and integration named conformable fractional derivative (CFD) and integral.

Definition 1.1. $f:[0, \infty) \rightarrow R$ be a function. The $\alpha^{\text {th }}$ order CFD of $f$ is defined by,

$$
D_{a}^{\alpha}(f)(t)=\lim _{\varepsilon \rightarrow 0} \frac{f\left(t+\varepsilon t^{1-\alpha}\right)-f(t)}{\varepsilon}
$$

for all $t>0, \alpha \in(0,1)$.

Definition 1.2. Let $f$ be $\alpha$ differentiable in some $(0, a), a>0$ and $\lim _{t \rightarrow 0^{+}} f^{(\alpha)}(t)$ exist then let $f^{(\alpha)}(0)=\lim _{t \rightarrow 0^{+}} f^{\alpha}(t)$. The conformable fractional integral of a function $f$ starting from $a \geq 0$ is mentioned as:

$$
I_{a}^{\alpha}(f)(t)=\int_{a}^{t} f(x) d_{\alpha} x=\int_{a}^{t} \frac{f(x)}{x^{1-\alpha}} d x
$$

that the integral denotes Riemann improper integral and $\alpha \in(0,1]$.

Following theorem [1] expresses some basic properties satisfied by CFD.

Theorem 1.1. Let $\alpha \in(0,1]$ and $f, g$ are $\alpha$ differentiable functions at point $t>0$, then

1. $T_{\alpha}(m f+n g)=m T_{\alpha}(f)+n T_{\alpha}(g)$ for all $m, n \in R$

2. $T_{\alpha}\left(t^{p}\right)=p t^{p-\alpha}$ for all $p$

3. $T_{\alpha}(f g)=f T_{\alpha}(g)+g T_{\alpha}(f)$ 
4. $T_{\alpha}\left(\frac{f}{g}\right)=\frac{g T_{\alpha}(f)-f T_{\alpha}(g)}{g^{2}}$

5. $T_{\alpha}(c)=0$ for all constant functions $f(t)=c$

6. Also, let $f$ be a differentiable function,
After existence of these definitions huge amount of applications are made by many scientists [15-26] In this work is going to shed some light on conformable time fractional KP equation [34]

then $T_{\alpha}(f)(t)=t^{1-\alpha} \frac{d f(t)}{d t}$.

$$
\frac{1}{4} \frac{\partial^{4} u}{\partial x^{4}}+\frac{3}{2} \frac{\partial u}{\partial x} \frac{\partial^{2} u}{\partial x^{2}}+\frac{3}{4} \frac{\partial^{2} u}{\partial y^{2}}+\frac{\partial}{\partial x} \frac{\partial^{\alpha}}{\partial t^{\alpha}}(u)=0
$$

The KP equation aroused as generalization of KDV equation [32] and used as a model for shallow long water waves. Also this equation is an integrable equation and used for modelling waves in ferromagnetic media [33].

\section{Basics of Sub-Equation Method}

In this section, we mention a short description of sub equation method [27]. Regard the conformable fractional partial differential equation (CFPDE) as

$$
P\left(u, D_{t}^{\alpha} u, D_{x} u, D_{y} u, D_{t}^{2 \alpha} u, D_{x}^{2} u, D_{y}^{2} u \ldots\right)=0
$$

where $D_{t}^{\alpha} u$ denotes CFD of $u(x, y, t)$ and $D_{t}^{2 \alpha} \quad u(x, y, t)$. By using the conformable wave shows two times CFD of the function transform [15]

$$
u(x, y, t)=U(\xi), \xi=m x+n y+p \frac{t^{\alpha}}{\alpha}
$$

and the chain rule [28] Eq. (2.1) becomes to a differential equation

$$
U(\xi)=\sum_{i=0}^{N} a_{i} \varphi^{i}(\xi), a_{N} \neq 0
$$

$G\left(U \cdot U^{\imath}, U^{u}, \ldots\right)=0$

In Eq. (2.3) prime indicates the derivative with respect to $\xi$. Also $m, n, p$ are arbitrary constants to be evaluated later. It is assumed that the solution of Eq. (2.3) can be given as

where $\sigma$ is a constant. Some of the solutions of Eq. (2.5) can be obtained as follows.

$$
\varphi^{\imath}(\xi)=\sigma+(\varphi(\xi))^{2}
$$

that the constants $a_{i}(0 \leq i \leq N)$ are going to be utilized. Also positive integer $N$ can be evaluated with the help of balancing solution of the equation

(2)
procedure [29] in Eq. (2.3) and $\varphi(\xi)$ is the 


$$
\phi(\xi)=\left\{\begin{array}{l}
-\sqrt{-\sigma} \tanh (\sqrt{-\sigma} \xi), \sigma<0 \\
-\sqrt{-\sigma} \operatorname{coth}(\sqrt{-\sigma} \xi), \sigma<0 \\
\sqrt{\sigma} \tan (\sqrt{\sigma} \xi), \sigma>0 \\
-\sqrt{\sigma} \cot (\sqrt{\sigma} \xi), \sigma>0 \\
-\frac{1}{\xi+\varpi}, \varpi \text { is a cons., } \sigma=0
\end{array}\right.
$$

Subrogating Eqs. (2.4) and (2.5) in Eq. (2.3), a polynomial of $\mathrm{t} \varphi(\xi)$ arises. Equating the coefficients of $\varphi^{i}(\xi)(i=0,1, \ldots, N)$ to zero led to an equation system with respect to $k, a_{i}(i=0,1, \ldots, N)$. Solving the system we obtain $k, p, a_{i}(i=0,1, \ldots, N)$. Subrogating the acquired values of the constants and the solution set of Eq. (2.5) into Eq. (2.4) with the aid of (2.6) we handle the analytical solutions for Eq. (2.1).

\section{Solution of Time Fractional KP wave transform (2.2) the Eq. (1.1) changes Equation}

Considering time fractional KP equation (1.1) then using the chain rule [28] and conformable

$$
\frac{1}{4} m^{4} U^{(v)}(\xi)+\frac{3}{2} m^{3} U^{\imath}(\xi) U^{u}(\xi)+\frac{3}{4} n^{2} U^{u}(\xi)+m p U^{u}(\xi)=0 .
$$

Integrating the above equation once and making some algebraic calculations led to

$$
m^{4} U^{u l}(\xi)+3 m^{3}\left(U^{\imath}(\xi)\right)^{2}+3 n^{2} U^{\imath}(\xi)+4 m p U^{\imath}(\xi)=0
$$

Assuming the solution of Eq. (3.2) is denoted where $\varphi(\xi)$ is the analytical solutions of by the following series

$U(\xi)=\sum_{i=0}^{N} a_{i} \phi^{i}(\xi), a_{N} \neq 0$ differential Eq. (2.5) given in (2.6). With the aid of balancing principle [29], we get $\mathrm{N}=1$. When substituting the obtained data into (3.1), led to an equation system.

$$
\begin{aligned}
& 3 a_{1}^{2} m^{3} \sigma^{2}+2 a_{1} m^{4} \sigma^{2}+4 a_{1} m p \sigma+3 a_{1} n^{2} \sigma=0, \\
& 6 a_{1}^{2} m^{3} \sigma+8 a_{1} m^{4} \sigma+4 a_{1} m p+3 a_{1} n^{2}=0, \\
& 3 a_{1}^{2} m^{3}+6 a_{1} m^{4}=0 .
\end{aligned}
$$

The solution set for system (3.4) can be expressed as

$$
a_{0}=a_{0}, a_{1}=-2 m, p=\frac{4 m^{4} \sigma-3 n^{2}}{4 m} .
$$




\section{For $\sigma>0$}

$$
\begin{aligned}
& u_{1}(x, y, t)=a_{0}+2 m \sqrt{-\sigma} \tanh \left(\sqrt{-\sigma}\left(m x+n y+\frac{4 m^{4} \sigma-3 n^{2}}{4 m} \frac{t^{\alpha}}{\alpha}\right)\right) \\
& u_{2}(x, y, t)=a_{0}+2 m \sqrt{-\sigma} \operatorname{coth}\left(\sqrt{-\sigma}\left(m x+n y+\frac{4 m^{4} \sigma-3 n^{2}}{4 m} \frac{t^{\alpha}}{\alpha}\right)\right)
\end{aligned}
$$

For $\sigma>0$

$$
\begin{aligned}
& u_{3}(x, t)=a_{0}-2 m \sqrt{\sigma} \tan \left(\sqrt{\sigma}\left(m x+n y+\frac{4 m^{4} \sigma-3 n^{2}}{4 m} \frac{t^{\alpha}}{\alpha}\right)\right), \\
& u_{4}(x, t)=a_{0}+2 m \sqrt{\sigma} \cot \left(\sqrt{\sigma}\left(m x+n y+\frac{4 m^{4} \sigma-3 n^{2}}{4 m} \frac{t^{\alpha}}{\alpha}\right)\right),
\end{aligned}
$$

For $\sigma=0$

$$
u_{5}(x, t)=a_{0}+\frac{2 m}{\varpi+m x+n y-\frac{3 n^{2}}{4 m} \frac{t^{\alpha}}{\alpha}} .
$$

Now let's give some graphical representations 4 show the solitary wave solution of the of obtained results. In Figure 1 represents the obtained results. travelling wave solution, Figures 2 and Figure

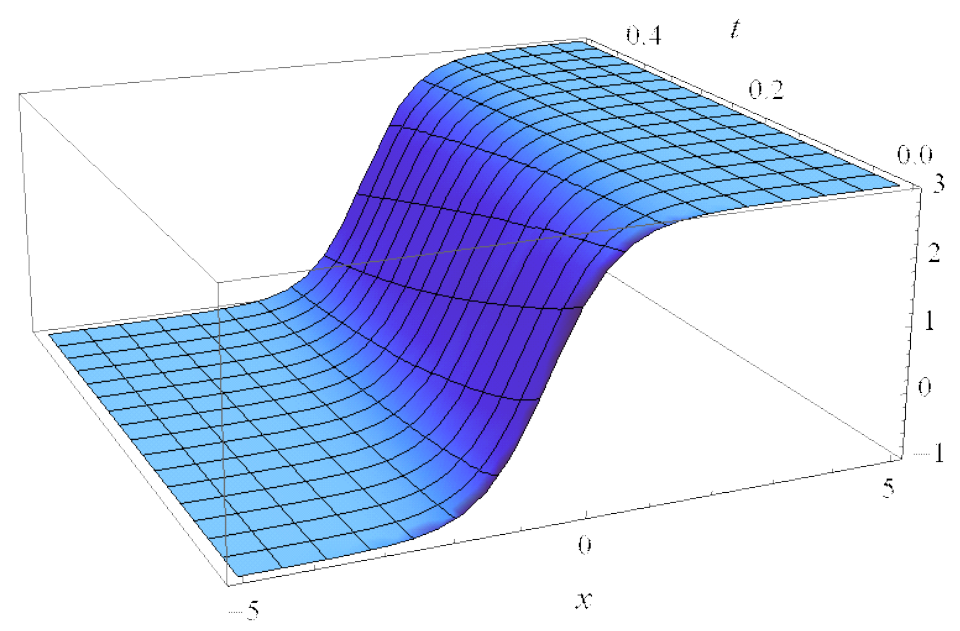

Figure 1: The surface plot of $u_{1}(x, y, t)$ for $m=1, n=1, \sigma=-1, a_{0}=1, \alpha=0.8, y=5$. 


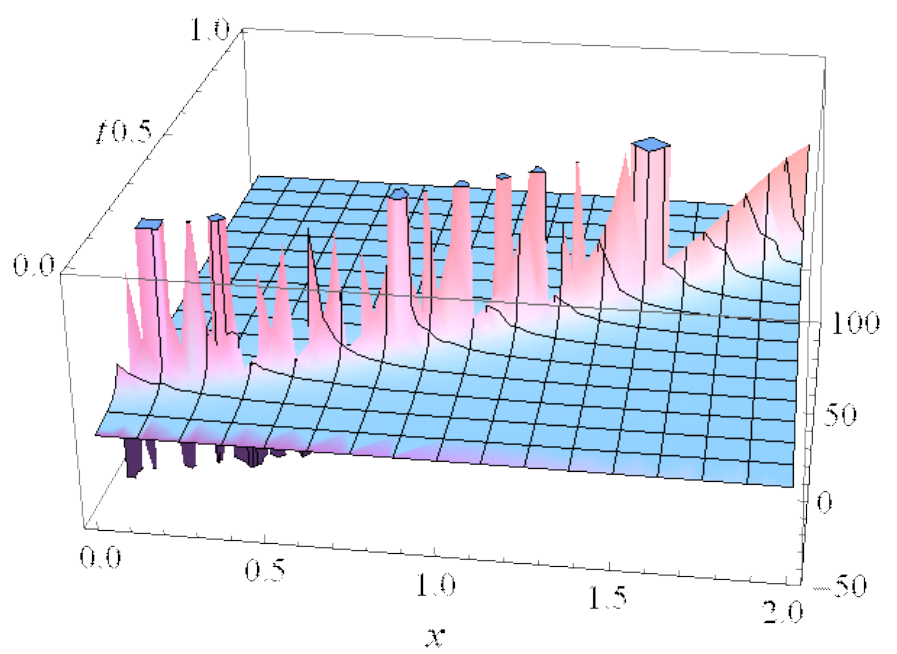

Figure 2: The graphical illustration of $u_{2}(x, y, t)$ for

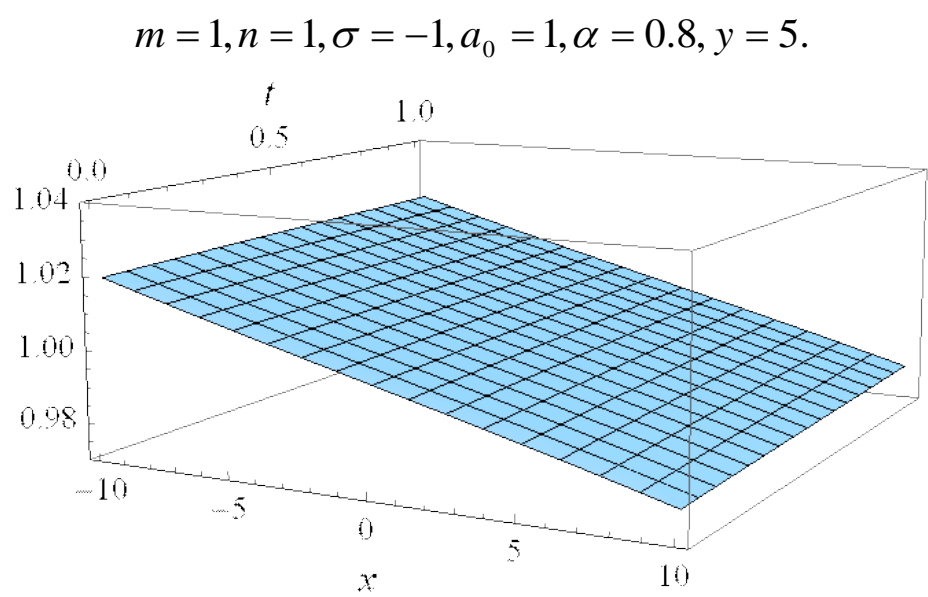

Figure 3: The graphical representation of $u_{3}(x, y, t)$ for

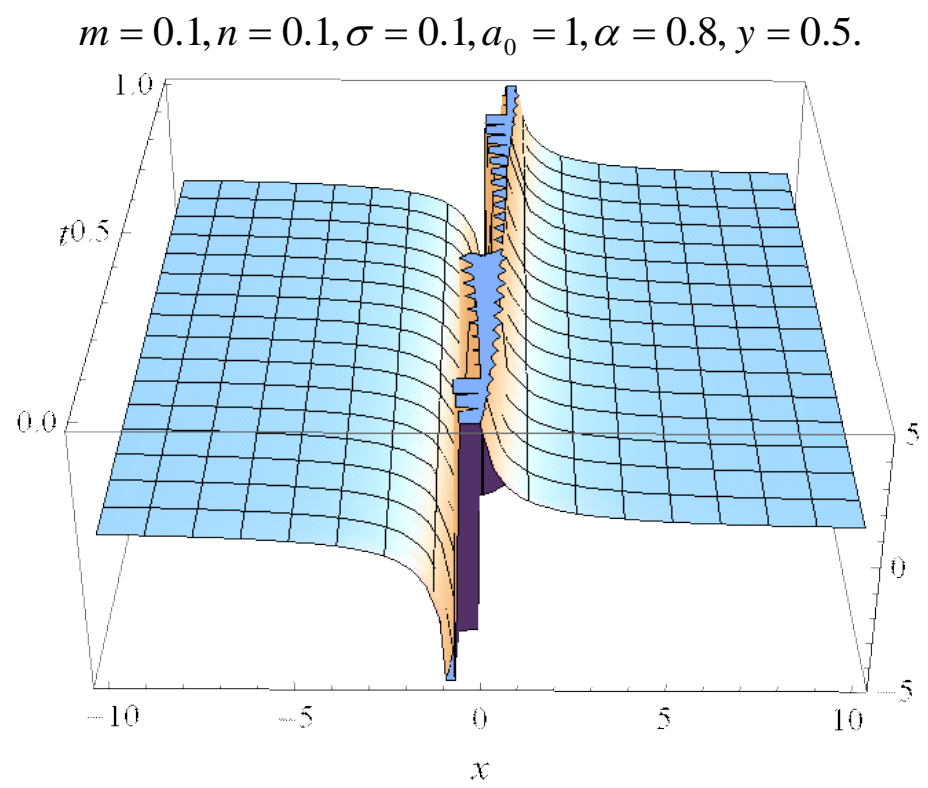

Figure 4: 3D graphical simulation of $u_{4}(x, y, t)$ for $m=0.1, n=0.1, \sigma=0.1, a_{0}=1, \alpha=0.8, y=0.5$. 


\section{Results}

The sub-equation method is employed to acquire the new exact solutions for the fractional KP equation. It is seen that the considered method is efficient and effective mathematical tool to handle conformable fractional nonlinear evolution equations that arises in many sciences. The CFD is well behaved and applicable derivative. Also this derivative has some advantages over other popular definitions. Especially obtaining the exact results of CFPDEs using the chain rule and wave transform is the most important property of CFD definition.

\section{References}

Khalil, R., Al Horani, M., Yousef, A., Sababheh, M., (2014), A new definition of fractional derivative, Journal of Computational and Applied Mathematics, 264, 65-70.

Abdeljawad, T., (2015), On conformable fractional calculus. Journal of computational and Applied Mathematics, 279, 57-66.

Kurt, A., Tasbozan, O., (2015)., Approximate Analytical Solution of the Time Fractional Whitham-Broer-Kaup Equation Using the Homotopy Analysis Method. International Jour-nal of Pure and Applied Mathematics, 98(4), 503-510.

Alquran, M., (2014), Analytical solutions of fractional foam drainage equation by residual power series method. Mathematical sciences, 8(4), 153-160.

El-Ajou, A., Arqub, O. A., Zhour, Z. A., Momani, S., (2013), New results on fractional power series: theories and applications. Entropy, 15(12), 5305-5323.

Jaradat, H. M., Al-Shara, S., Khan, Q. J., Alquran, M., Al-Khaled, K., (2016), Analytical solution of time-fractional Drinfeld-Sokolov-Wilson system using residual power series method. IAENG Int. J. Appl. Math, 46(1), 64-70.

Kumar, A., Kumar, S., Singh, M., (2016), Residual power series method for fractional Sharma-Tasso-Olever equation. Communications in Numerical Analysis, 2016(1), 1-10.

Senol, M., Kasmaei, H. D., (2017), On the Comparison of Perturbation-Iteration Algorithm and Residual Power Series Method to Solve Fractional Zakharov-Kuznetsov Equation. arXiv preprint arXiv:1708.07970.

Li, Z., (2014), Heteroclinic Breather-Wave for the Coupled Schrdinger Boussinesq Equation. Applied Mathematical Sciences, 8(120), 5995-6000.

Chowdhury, A. R., Rao, N. N., (1998), Painlve Analysis and Backlund Transformations for Coupled Generalized Schrdinger Boussinesq System. Chaos, Solitons and Fractals, 9(10), 1747-1753.

Huang, X., (2013), The investigation of solutions to the coupled SchrödingerBoussinesq equations. Abstract and Applied Analysis (Vol. 2013). Hindawi.

Zayed, E. M. E., Alurr, K. A. E., (2014), On solving the nonlinear Schrödinger-Boussinesq equation and the hyperbolic Schrödinger equation by using the $\left(G^{\prime} / G\right)$ expansion method. International Journal of Physical Sciences, 9(19), 415-429.

Khater, M. M., Kumar, D., (2017), Implementation of three reliable methods for nding the exact solutions of $(2+1)$ dimensional generalized fractional evolution equations. Optical and Quantum Electronics, 49(12), 427.

Kaplan, M., Bekir, A., (2016), A novel analytical method for time-fractional differential equations. Optik-International Journal for Light and Electron Optics, 127(20), 8209-8214.

Cenesiz, Y., A. Kurt., (2016), New fractional complex transform for conformable fractional 
partial differential equations." Journal of Applied Mathematics, Statistics and Informatics, 12, 41-47.

Korkmaz, A., (2017), Exact solutions to (3+1) conformable time fractional Jimbo-MiwaZakharov-Kuznetsov and modified ZakharovKuznetsov equations. Communications in Theoretical Physics, 67(5), 479.

Korkmaz, A., Hosseini, K., (2017), Exact solutions of a nonlinear conformable timefractional parabolic equation with exponential nonlinearity using reliable methods. Optical and Quantum Electronics, 49(8), 278.

Cenesiz, Y., Baleanu, D., Kurt, A., Tasbozan, O., (2017), New exact solutions of Burgers type equations with conformable derivative. Waves in Random and Complex Media, 27(1), 103-116.

Kurt, A., Tasbozan, O., Baleanu, D., (2017), New solutions for conformable fractional Nizhnik-Novikov-Veselov system via $\left(G^{\prime} / G\right)$-expansion method and homotopy analysis methods. Optical and Quantum Electronics, 49(10), 333.

Hosseini, K., Mayeli, P., Ansari, R., (2017), Bright and singular soliton solutions of the conformable time-fractional Klein \{Gordon equations with different nonlinearities. Waves in Random and Complex Media, 1-9.

Rezazadeh, H., Manafian, J., Khodadad, F. S., Nazari, F., (2018), Traveling wave solutions for density-dependent conformable fractional diffusion-reaction equation by the first integral method and the improved $\tan \left(\frac{1}{2} \varphi(\xi)\right)$-expansion method. Optical and Quantum Electronics, 50(3), 121.

Eslami, M., Khodadad, F. S., Nazari, F., Rezazadeh, H., (2017), The first integral method applied to the Bogoyavlenskii equations by means of conformable fractional derivative. Optical and Quantum Electronics, 49(12), 391.
Khodadad, F. S., Nazari, F., Eslami, M., Rezazadeh, H., (2017), Soliton solutions of the conformable fractional ZakharovKuznetsov equation with dual-power law nonlinearity. Optical and Quantum Electronics, 49(11), 384.

Eslami, M., Rezazadeh, H., Rezazadeh, M., Mosavi, S. S., (2017), Exact solutions to the space-time fractional Schrdinger-Hirota equation and the space-time modified KDVZakharov Kuznetsov equation. Optical and Quantum Electronics, 49(8), 279.

Eslami, M., Rezazadeh, H., (2016), The first integral method for Wu-Zhang system with conformable time-fractional derivative. Calcolo, 53(3), 475-485.

Aminikhah, H., Sheikhani, A. R., Rezazadeh, H., (2016), Sub-equation method for the fractional regularized long-wave equations with conformable fractional derivatives. Scientia Iranica. Transaction B, Mechanical Engineering, 23(3), 1048.

Zhang, Sheng, and Hong-Qing Zhang., (2011), Fractional sub-equation method and its applications to nonlinear fractional PDEs", Physics Letters A, 375.7(2011), 1069-1073.

Abdeljawad T., (2015), On conformable fractional calulus, J. Comput. Appl. Math., 279, 57-66.

Malfiet, W, (1992), Solitary wave solutions of nonlinear wave equations, American Journal of Physics 60, 650-654.

Ali, M. N., Husnine, S. M., Ak, T., Atangana, A. (2018), Solitary wave solution and conservation laws of higher dimensional Zakharov-Kuznetsov equation with nonlinear self-adjointness, Mathematical Methods in the Applied Sciences 41(16), 6611-6624.

Dokuyucu, M. A., Celik, E., Bulut, H., Baskonus, H. M., (2018), Cancer treatment model with the Caputo-Fabrizio fractional derivative. The European Physical Journal Plus 133(3), 92. 
Kadomtsev, B. B., Petviashvili, V. I., (1970), On the stability of solitary waves in weakly dispersive media, Sov. Phys. Dokl. 15, 539541.

Wazwaz, A. M., (2007), Multiple-soliton solutions for the KP equation by Hirota's bilinear method and by the tanh-coth method, Applied Mathematics and Computation, 190(1), 633-640.

Bekir, A., Guner, O., (2014), Exact solutions of distinct physical structures to the fractional potential Kadomtsev-Petviashvili equation, Computational Methods for Differential Equations 2(1), 26-36. 